\title{
THE HORMONAL CHANGES AMONG NIGERIAN WOMEN WITH EPILEPSY
}

Osalusi, Bamidele Sanya*; Ogunjimi, Lukman; Yaria, Joseph; Ale, Ayotunde; Ogunsemi, Olawale; Akinyinka, Alabi; Ogunniyi, Adesola

Department of Medicine, Obafemi Awolowo College of Medicine, Olabisi Onabanjo University

Email - sanyabamidele@yahoo.com

\section{ABSTRACT}

This study aims to compare the sex hormones between women with epilepsy (WWE) and their agehormonal profile. A case control study carried out at the University College Hospital, Ibadan South western, Nigeria involving seventy-five WWE and age-matched controls. Blood samples for hormonal evaluation follicle stimulating hormone (FSH), luteinizing hormone (LH), estrogen, progesterone and testosterone were taken twice from all the participants during their menstrual cycle. WWE had lower BMI (p: 0.004), cycle length ( $\mathrm{p}: 0.014$ ) and more menstrual pattern irregularities ( $\mathrm{p}<$ 0.001 .) In the pre-ovulatory phase, WWE had lower FSH levels when compared with controls, (p: $0.012)$. Further stratification shows a higher FSH levels among WWE on medication, (p: 0.003).In the mid-luteal phase, FSH level was lowest in WWE not on medication, WWE on medication had higher

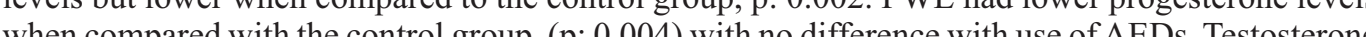
when compared with the control group, (p: 0.004) with no difference with use of AEDs. Testosterone low FSH ond morenes

Keywords: Hormonal Changes, Women, Epilepsy, Nigeria

Accepted Date:15 May, 2020

\section{INTRODUCTION}

The regular and synchronized release of various sex hormones ensures oocyte maturation, ovulation, and menstruation (Velíšková et al, 2010, Harden \& Pennell 2013) Due to the cyclical nature of hormone release and the direct neuronal effects of oestrogen and progesterone or their metabolites, women are especially susceptible to the effects of these shifting hormones onstible to the effects of severity (Velišková et al, 2010, Velíšková \& DeSantis 2013). Thehormonal effects not only causes rapid changes in neuronal activity (Reddy 2013) but also long term changes from involvement of intracellular receptors that mediates gene expression at the nuclear level(Velíšková et al 2010, Harden \& Pennell 2013).The relationship between epilepsy and sex steroid hormones has been explored with studies showing a bi-directional complex interdependence(Velíšková et al, 2010 Velíśková \& DeSantis 2013). Progesterone has long been shown in several studies to have antiseizure activities (Herzog et al. 2015, Younus \&
Reddy 2017 Pennell 2009). Reports have also postulated that progester one is the basis behind a lower incidence of epilepsy in women than men (Di Mai 2014). To this effect, progesterone has been linked to management of a number of brain related conditions e.g. traumatic brain injury (Harden \& Pennell 2013 Velíšková \& DeSantis 2013, Harden et al. 2006). Estrogen on the other hand is believed to have pro-convulsant and epileptogenic property (Sato \& Woolley 2016. Velísková 2000) Intravenous infusions of estrogen is associated with rapid inter-ictal epilepti form activity in women with epilepsy (WWE), and seizures were exacerbated when estrogen was given prior to menstruation (Harden et al., 2006 Luef 2010). However, catamenial epilevsy clinic is an uncomiteps in our routine pilepsy clinic is an uncono (Hoccurrence despite prevalence as high as $78 \%$ (H Herzog 2015). Also, result of an earlier study that showed no difference in the frequency of clinically significant sexual dysfunction between women with epilepsy (WWE) and their matched controls in 
our cohort informed this study (Ogunjimi et al., 2018). This study aims to compare the sex hormones between WWE and their age-matched controls. We postulated that a difference in etiology, with a higher prevalence of structural and/or acquired epilepsy in sub-Saharan Africa (Ba-Diop et al., 2014, Preux \& Druet-Cabanac 2005), may be associated with an unexpected hormonal profile.

\section{MATERIALS AND METHODS}

We conducted an observational study at the Neurology clinic, University College Hospital, Nigeria involving seventy-five WWE and fortyfive controls of comparable ages between August 2015 and august 2016. The diagnosis of epilepsy was made clinically with electro encephalographic (EEG) features taken into consideration. Participants with a background chronic medical illness, features suggestive of pseudo-seizures, on oral contraceptives, pre-diagnosis of hypogonadism, structural gynecological anomalies, amenorrhea and those on hormonal replacement therapy were excluded. Ethical approval was obtained from the Joint Institution Review Committee (IRC) of the University College Hospital and the College of Medicine, University of Ibadan, Nigeria An interviewer administered questionaire administered by trained Neurlogist was used to obtain socio-demographic variables and relevant clinical information from recruited participants which include epilepsy frequency, semiology, etiology and anti-epileptic drugs (AED) use. A Phoenix digital 16-channel EEG was used to obtain tracing in all participants. EEG recording lasted at least 45 minutes, with activation procedures including hyperventilation and photic stimulation. Detailed hospital records and medical history with focus on menstrual cycle characteristi controls.
A regular menstrual cycle was taken as 21-34 day duration (Amu \& Bamidele 2014.). Blood samples for hormonal evaluation-follicle stimulating hormone (FSH), luteinizing hormone (LH) estrogen, progesterone and testosterone were taken from all participants twice during their menstrual cycle. The first sample was th ovulatory phase on The cycle. The second sample was withdrawn during the luteal phase on the $21^{\text {st }}-24^{\text {th }}$ day. Enzyme linked immune-sorbent assay technigue was used to determine the hormonal concentration. Dat obtained were entered in Microsoft Excel for cleaning and subsequently transferred to Stat version 12 for analysis. The Pearson Chi-square or Fisher's exact (where the expected counts were les Five per cell) was used to assess categorica tariale. Wilcoxon rank-sim and Kruskal Wallis test was used to determine continuous variable. The level of significance was $\mathrm{P}<.005$.

\section{RESULTS}

The ages of participants ranged from 16-57 years with mean age of WWE and controls, 32.1 years $(\mathrm{SD}: \pm 9.3$ years) and 31.2 years (SD: \pm 6.7 years) respectively; (t: -0.56 , p-value: 0.575 .) There was a significant difference in mean body mass index signific between bo a among WWE as opposed to $27.5 \mathrm{~kg} / \mathrm{m} 2$ (SD: \pm 5 . $\mathrm{kg} / \mathrm{m} 2$ ) among controls; t: 2.97, p-value: 0.004 WWE and controls had similar gynecologica features with respect to age at menarche (p: 0.959) bleeding days (p: 0.347) and frequency of dysmenorrhea (p: 0.072) but differ in cycle length and menstrual pattern with WWE having shorte cycle length (p: 0.014). The prevalence of menstrual disturbence among WWE in our cohot was $39.7 \%$. Sixty p pert $(60.3 \%)$ cohor admitted to normal menstrual flow as opposed to $97.5 \%$ controls $(\mathrm{p}<0.001)$ with $18(24.6 \%)$, and 1 $(15.1 \%)$ WWE admitting to hypo-menorrhea, and hyper-menorrhea. See Table .
Table 1: Menstrual Related Characteristics

\begin{tabular}{lllll} 
& WWE & Controls & Stat & p-value \\
\hline Age, Mean (SD) & $32.1(9.3)$ & $31.2(6.7)$ & -0.56 & 0.575 \\
BMI, Mean (SD) & $24.7(4.6)$ & $27.5(5.7)$ & 12.97 & 0.004 \\
Menarche, Mean (SD) & $12.7(1.9)$ & $12.7(1.8)$ & 10.05 & 0.959 \\
Bleeding Days, Mean (SD) & $4.7(0.8)$ & $4.9(1.2)$ & ${ }^{1} 0.95$ & 0.347 \\
Dysmenorrhea, N (\%) & $46(61.3)$ & $20(44.4)$ & ${ }^{2} 3.24$ & 0.072 \\
Cycle Length, Mean (SD) & $27.1(4.0)$ & $28.9(5.0)$ & ${ }^{3} 2.46$ & 0.014 \\
Normal Menstrual Flow, N (\%) & $44(60.3)$ & $39(97.5)$ & ${ }^{2} 18.58$ & $<0.001$ \\
\hline${ }^{\prime}$ Independent student t-test (t-statistic). ${ }^{2}$ Pearson Chi-square test $\left(\mathrm{X}^{2}\right){ }^{3}$ Mann Whitney test (Z-score)
\end{tabular}

Among WWE, those on Anti epileptic Drugs (AED) had a shorter cycle length, 26.6 ( \pm 3.6$)$ days; bleeding days, $4.6( \pm 0.8)$ days; and a higher frequency of dysmenorrhea, $40(69.0 \%)$ when compared to those not on AED who had cycle length, $30.1( \pm 5.3)$ days, bleeding days, $5.3( \pm 0.7)$ days, and dysmenorrhea frequency of $6(35.3 \%)$, p-values: $p: 0.021,0.031$ and 0.012 respective Using the 2017 Intern pe Against Epilepsy classification, of the studied subjects $38(50.7 \%)$ had combined, $18(24.0 \%)$ had generalized, $12(16.0 \%)$ had focal unaware, and 7 (9.3\%) had focal aware epilepsy. Of the $75 \mathrm{WWE}$; $39(52.0 \%)$ had structural, $30(40.0 \%)$ had idiopathic, 5 (6.7\%) had infective, and one (1.3\%) metabolic etiology. Concerning treatment, 58 (77.3\%) were on AED - carbamazepine, all within 2 years since commencement, with 7 out of 58 (12.1\%) admitting to poor medication adher 58

control, there was no statistical difference in seizure control between WWE not on medication and WWE on medication, $X^{2}: 1.99$, p: 0.159.

In the pre-ovulatory phase, WWE had lower FSH levels $(5.8 \mathrm{mIU} / \mathrm{ml}$; IQR: $3.2-11.7 \mathrm{mIU} / \mathrm{ml})$ when compared with controls $(8.3 \mathrm{mIU} / \mathrm{ml}$; IQR: 6.3 $13.0 \mathrm{mIU} / \mathrm{ml}$ ), Z: $2.518, \mathrm{p}: 0.012$. Furthe stratification shows that WWE on 0.012 . Further higher FSH levels $(6.4 \mathrm{mIU} / \mathrm{m}$ $\mathrm{mIU} / \mathrm{ml}$ ) when compared to WWE not on treatment (4.6 mIU/ml; IQR: $2.3-5.5 \mathrm{mIU} / \mathrm{ml})$, p: 0.003 Controls had similar LH/FSH ratio with WWE on medication. However, WWE not on medication had a higher LH/FSH ratio $(1.3 \mathrm{mIU} / \mathrm{ml}$; IQR: 0.7 $10.3 \mathrm{mIU} / \mathrm{ml}$ ) when compared to WWE on medication $(0.9 \mathrm{mIU} / \mathrm{ml}$; IQR: $0.5-1.1 \mathrm{mIU} / \mathrm{ml})$. There was no significant difference in the levels of WWE with or without treatment. see Table2.

\begin{tabular}{lllll}
\multicolumn{5}{l}{ Table 2: Pre-ovulatory Phase Hormonal level } \\
& $\begin{array}{l}\text { WWE not on } \\
\text { AEDs } \\
\text { mIU/ml, Med }\end{array}$ & $\begin{array}{l}\text { WWE on AEDs } \\
\text { mIU/ml, Med } \\
(\mathrm{IQR})\end{array}$ & $\begin{array}{l}\text { Controls } \\
\mathrm{mIU} / \mathrm{ml} \text {, Med } \\
(\mathrm{IQR})\end{array}$ & $\mathrm{p}$ \\
& $4.6(2.3-5.5)$ & $6.4(3.7-14.2)$ & $8.3(6.4-13.0)$ & $<0.001$ \\
\hline FSH & $6.6(4.6-41.0)$ & $6.8(4.7-16.3)$ & $8.0(6.2-10.3)$ & 0.984 \\
LH & $1.3(0.7-10.3)$ & $0.9(0.5-1.1)$ & $0.9(0.6-1.2)$ & 0.026 \\
LH/FSH & $80.9(53.7-280.0)$ & $74.6(44.6-164.8)$ & $80.5(60-104.4)$ & 0.673 \\
Estrogen & $1.0(0.3-10.0)$ & $0.7(0.4-2.4)$ & $0.9(0.5-1.8)$ & 0.438 \\
Progesterone & & & \\
E/P ratio & $64.6(21.9-142.0)$ & $88.0(54-248.2)$ & $71.4(47.9-124.1)$ & 0.461 \\
\hline
\end{tabular}


In the mid-luteal phase, FSH level was lowest in WWE not on medication (4 mIU/ml; IQR: 1.3- 6.2 $\mathrm{mIU} / \mathrm{ml})$, FWE on medication had higher levels (5.6 mIU/ml; IQR: $3.2-11.0 \mathrm{mIU} / \mathrm{ml}$ ) but lower when compared to the control group $(8.3 \mathrm{mIU} / \mathrm{ml}$; IQR: $4.6-12.0 \mathrm{mIU} / \mathrm{ml})$, p: 0.002 . WWE on medication and those without however had similar progesterone level which was significantly lower than levels among the control group, (X2.10.9, p: $0.004)$. The E/P ratio was higher in WWE when $0.004)$ The E/P rio was highe compared to conts and anong the WWE, those on medication had similar values compared to those without medications (X2: 13.0, p: 0.002). There was no significant difference in the levels of $\mathrm{LH}, \mathrm{LH} / \mathrm{FSH}$ ratio, estrogen, E/P ratio, and testosterone between WWE and controls levels of LH, FSH, progesterone and estrogen did not vary with epilepsy classification and were comparable in patients with idiopathic epilepsy and those with symptomatic epilepsy. However, testosterone levels were lower among those with symptomatic epilepsy (2.4 mlU/ml; IQR: 1.0-3.8 $\mathrm{mlU} / \mathrm{ml})$ when compared to those with idiopathic epilepsy (1.1 $\mathrm{mIU} / \mathrm{ml}$; IQR: $0.7-1.7 \mathrm{mIU} / \mathrm{ml})$; p-value: 0.012 . This occurs despite no difference in clinical outcome seen in the use of carbamazepine between both groups ( $73.3 \%$ vs. $76.9 \%)$

\section{DISCUSSION}

The lower proportions of abnormal menstrual patterns in controls compared to WWE suggest an under lying hormonal abnormality among WWE and underscores the need to evaluate the effect of epilepsy and AEDs on menstrual cycles among women. Interestingly, the prevalence of menstrual disturbance among WWE in our cohort was similar to results from other studies (Amu \& Bamidele 2014. Svalheim et al., 2003). In this study, WWE had lower FSH values with even lower score among WWE without medications in both preovulatory and luteal phase similar to findings from a previous study by El- Khayat et al (2008). Possible explanation include; the negative effect of epilepsy on ovulation and menstruation, effect of epilepsy on ovulation and menstruation, effect of
carbamazepine, or seizure effect on the hypothalamus. Left temporallobe epilepsy has been shown to have an effect on FSH and this can explain lower levels in WWE. The concomitant increase in FSH level among WWE on carbamazepine raises the possibility of reduction or resolution of seizure events as the mechanism behind his change in FSH level. This may however be unlikely in this study as there was no change in seizure Control between FWE on medications an those not on medication. The relationship between menstrual parameters and AED in the study will suggest carbamazepine may act at this level. Carbamazepine has been shown to impact on FSH level, as it aids conversion of inactive to active metabolite(Bangar et al.2016, Svalheim et al. 2009 , Verrotti et al. 2011) The fact that an syla syndrome is less commen polycystic ovarian contrary to the use of other anti-epileptic drugs that reduces the FSH level also supports this notion (Bangar et al., 2016; Svalheim et a l., 2009, Verrott et al. 2011). It is therefore explainable that values of LH/FSH among WWE not on carbamazepine were greater than 1 in our study. Notably, Miia et al, ( 2006) demonstrated the differential effect of carbamazepine on LH, FSH prolactin, sex hormone binding globulin at two months and twelve months respectively. Further studies to ascertain the acute, chronic and cytochrome p450 activities of carbamazepine action on sex steroid and ovulation in FWE with focus pharmacogenomics is suggested. The fact that WWE had similar estradio levels with controls in this study is surprising. With themetabolic effect of AEDs on estradiol, a lowe estradiol is expected. Also, with a lower FSH level in WWE, a lower estradiol can be expected. However, we postulate that peripheral action of cirion to higher estadio than expected. This couple with increase estradiol receptor sensitivity in the brain of WWE (Verrotti et al., 2009, Viswanathan et al., 2016, Mukherjee et al., 2017) can both explain the lower FSH seen in WWE, change in menstrual characteristics and absence of clinically relevant sexual dysfunction earlier documented (Ogunjim et al. 2018). Another explanation not explored in this study is the acute versus chronic seizure effect and pulsatility of gonadotopins. Moreclinicald cal dat are emerging that estrogen may have no proconvulsant or even anticonvulsant effects, even though it is commonly accepted that estroge affects neuronal excitability and thus may mediate pro-convulsant effects (Preux \& Druet-Cabanac 2005). The pro-convulsant effect of estrogen has been linked to modulation of gene expression, direct interaction with neurotransmitter receptors and release and induction of brain derived neurotrophic factor (BNDF) which potentiates excitatory glutaminergic pathway in the hippocampus and elevation in seizure frequency, particularly after estradiol surge during periovulatory catamenial epilepsy (Scharfman \& MacLusky 2006,Sohrabji et al 1995, Wang et al., 2018). However, estrogen also closely relate to endogenous molecules like NPY with seizure protecting property. There is a sequential induction of BDNF and NPY to bring about transient rise and all in seizures frequency (Scharfman \& MacLusky 2006. Scharfman \& MacLusky 2008). Again, as demonstrated in this study, WWE had a lower progesterone level in the luteal phase and by extension a higher $\mathrm{E} / \mathrm{P}$ ratio. This is similar to what was reported by various authors (Velíšková et al 2010 Mukherjee et al., 2017; Scharfman \& MacLusky 2008). Interestingly, the peri-ovulatory pattern $\mathrm{C} 1$, of Catamenial epilepsy has been best explained by high E/P ratio (Harden \& Pennell 2013, Herzog 2015, El Khayat et al., 2008). However, while a high E/P ratio is seen in this study, none of the FEW reported features suggestive of catamenial epilepsy. This may give credence to other possible explanation which includes rise in glucocorticoid levels, LH-FSH surge, sequential induction of brain derived growth factor (BDNF) and neuro peptide Y (NPY). The overall effect of estrogen however depends on treatment duration, mode of administration, seizure type/model, estrogendose and hormonal status.

It should be stated that the levels of LH, FSH, progesterone and estrogen did not vary with epilepsy classification and were comparable in patients with idiopathic epilepsy and those with symptomatic epilepsy. However, testosterone level was lower among those with symptomatic epilepsy even though use of carbamazepine was similar between both groups. The lower levels of testosterone in the female with symptomatic epilepsy compare th further cinforce our int fifference in etiology may be thought that a difference in etiology may be associated with an unexpected hormonal profile and therefore difference in clinical presentation.

Studies have suggested that epileptogenic focus has some association with hormonal profile. Epilepsy, especially those with involvement of limbic system might have ictal and post ictal effect on hypothalamo-pituitary-ovarian axis thus affecting the hormonal levels. Of importance however is tha temporal lobe epilepsy in particular have adverse effect on testicular endocrine function and unilateral amygadala seizures activates the hypothalamus in a laterally asymmetric fashion resulting in different endocrine disorders(Taubøll et al 2015).The left temporal lobe epilepsy was associated with significantly higher pulse frequency of gonadotrophin release hormone frect secretion which is associated with highe testosterone and LH/FSH ratio(Herzog et al. 2003 Herzog 1993). On the contrary, right temporal lobe epilepsy leads to a reduced GnRH pulse frequency, higher frequency of hypothalamic amenorrhea, lower LH and estradiol (Herzog et al., 2003). This may thus explain why both hypermenorrhea and hypomenorrhea are commoner in FWE compared with controls in this study and emphasizes the need for neuroimaging, the lack of which limits further analysis in this study. Also, evaluation of the interictal and post-ictal secretion of luteinizing hormone in mesial temporal lobe epilepsy revealed that acute seizures induced timing irregularity in luteinizing hormone secretion, whereas chronic epilepsy was associated with changes in luteinizing hormone pulse frequency, amplitude, and mass(Luef 2010; Herzog 1993). Our study demonstrated a relatively higher E/P, lower FSH and lower progesterone in the luteal phase among WWE with a higher prevalence of menstrual abnormalities.

\section{CONCLUSION}

The use of carbamazepine however, had an effect on menstrual symptoms and improved FSH levels. Also, testosterone levels were lower in the female with symptomatic epilepsy when compared to those with idiopathic epilepsy. A difference in seizure etiology may explain the unexpected hormonal profile seen in our setting. Therefore, physician should not only consider effect and the etiological when duration and lateralization of epilepsy, when managing females with epilepsy to optimize care and improve quality of life. The inability to properly localize epileptogenic focus is however limitation in the study as variations in hormone level cannot be fully explaine. 


\section{References}

Amu EO, Bamidele JO (2014 ). Prevalence of menstrual disorders among adolescent girls in Osogbo, South Western Nigeria. Int JAdolesc Med Health; 26: 101-106.

Bangar S, Shastri A, El-Sayeh H, et al (2016). Women with epilepsy: clinically relevant issues. Funct Neurol, 31:127-134.

Ba-Diop A, Marin B, Druet-Cabanac M, et al. (2014). Epidemiology, causes, and reatment of epilepsy in sub-Saharan Africa. Lancet Neurol, 13:1029-1044.

Di Maio R. Neuronal mechanisms of epileptogenesis (2014). Front Cell Neurosci, 8: 29.

El-Khayat HA, Soliman NA, Tomoum HY, et al (2008). Reproductive hormonal changes and catamenial pattern in adolescent females with epilepsy. Epilepsia, 49:16191626.

Harden CL, Pennell PB(2013) . Neuroendocrine considerations in the treatment of men and women with epilepsy,. Lancet Neurol, 12: $72-83$

Harden CL, Herzog AG, Nikolov BG, et al (2006). Hormone replacement therapy in women with epilepsy: a randomized, double-blind, placebo-controlled study. Epilepsia, 47 1447-1451.

Herzog AG, Fowler KM, Sperling MR, et al (2015). Distribution of seizures across the menstrual cycle in women with epilepsy. Epilepsia, 56: 58-62.

Herzog AG (2015). Catamenial epilepsy: Update on prevalence, pathophysiology and treatment from the findings of the NIH Progesterone Treatment Trial, Seizure, 28: 18-25 Herzog AG, Coleman AE, Jacobs $\mathrm{AR}$, et al (2003). Interictal EEG discharges, reproductive hormones and menstrual disorders in epilepsy. Ann Neurol,; 54:625-637.

Herzog AG(1993). A relationship between particular reproductive endocrine disorders and the laterality of epileptiform disorcers and Neurologe,43, 1907-1910. Neurology, 43, 1907-1910.

Luef $\mathrm{G}(2010)$. Hormonal alterations following seizures.Epilepsy Behav EB,19:131-133.
Miia Artama, J.I.T. Isojärvi, A. Auvinen (2006) Antiepileptic drug use and birth rate in patients with epilepsya population-based cohort study in Finland, Huma Reproduction, 21,(9): 2290-2295,

Mukherjee J, Cardarelli RA, Cantaut-Belarif Y, et al (2017). Estradiol modulates the efficacy of synaptic inhibition by decreasing the dwell time of GABAA receptors inhibitory synapses Proc NatAcctors at inhibitory synapses. Proc Natl Acad SciUS

Ogunjimi L, Yaria J, Makanjuola A, et al (2018) Sexual dysfunction among Nigerian women with epilepsy. Epilepsy Behav, 83: 108-112.

Preux P-M, Druet-Cabanac M.(2005) Epidemiology and aetiology of epilepsy in sub-Saharan Africa,

Lancet Neurol, 4:21-31Pennell PB(2009). Hormonal Aspects of Epilepsy. Neuro Clin; 27: 941.

Quigg M, Kiely JM, Shneker B, et al (2002).Interictal and postictal alterations of pulsatile secretions of luteinizing hormone in temporal lobe epilepsy in men. Ann Neurol, 51:559-566.

Reddy DS(2013). Role of hormones and neurosteroids in epileptogenesis. Front Cell Neurosci; 7:115.

Scharfman HE, MacLusky NJ.(2006) The Influence of Gonadal Hormones on Neuronal Excitability, Seizures, an Epilepsy in the Female. Epilepsia, 47, 1423-1440.

Sohrabji F, Miranda RC, Toran-Allerand CD(1995). Identification of a putative estrogen response element in the gene encoding brain-derived neurotrophic factor. Proc Natl Acad Sci U S A, 92, 11110-11114

Svalheim S, Taubøll E, Bjørnenak T, et al (2003). Do women with epilepsy have increased frequency of menstrual disturbances? Seizure; 12: 529-533

Svalheim S, Taubøll E, Luef G, et al (2009). Differential effects of levetiracetam, carbamazepine, and lamotrigine on reproductive endocrine function in adult reproductive endocrine function in adults
EpilepsyBehav EB;16:281-287 Scharfman HE, MacLusky NJ(2008).
Estrogen-Growth Factor Interactions and Their Contributions to Neurological Disorders. Headache; 48: S77-S89.

Sato SM, Woolley CS(2016). Acute inhibition of neurosteroid estrogen synthesis suppresses status epilepticus in an animal model. eLife; 5. Epub a head of print 15 . DOI. 10.7554/eLife.12917.

Taubøll E, Sveberg L, Svalheim S (2015) Interactions between hormones and epilepsy. Seizure, 28: 3-11.

Velíšková J, De Jesus G, Kaur R, et al (2010). Females, their estrogens and seizures. Epilepsia; 51:141-144.

Velišková J, DeSantis KA (2013). Sex and Hormonal influences on Seizures and Epilepsy. Horm Behav, 63: 267-277.

Velísková J, Velísek L, Galanopoulou AS, et a (000). Neuroprotective effects of estrogens on hippocampal cells in adult female rat after status epilepticus. Epilepsia, 241
Suppl 6, S30-35

Verrotti A, D'Egidio C, Coppola G, et al (2009). Epilepsy, sex hormones and antiepileptic drugs in female patients. Expert Rev Neurother, 9:1803-1814.

Verrotti A, D'Egidio C, Mohn A, et al(2011) Antiepileptic drugs, sex hormones, and PCOS. Epilepsia,; 52: 199-211.

Viswanathan LG, Satishchandra P, Bhimani BC, et al (2016). Polycystic ovary syndrome in patients on antiepileptic drugs. Ann Indian Acad Neurol, 19:339-343.

Wang Y-J, Chen K-Y, Kuo L-N, et al (2018). The association between BDNF Val66Met polymorphism and emotional symptoms after mild traumatic brain injury. BMC Med Genet; 19: 13 .

Younus I, Reddy DS2017. Epigenetic interventions for epileptogenesis: A new frontier for curing epilepsy. Pharmacol Ther, 177,10822 